\title{
Modelo predictivo de la importancia y utilidad de la Educación Física
}

\section{Predictive model of the importance and usefulness of Physical Education}

\section{Modelo preditivo da importância e utilidade da Educação Física}

\author{
A. Baena-Extremera ${ }^{1}$, A. Granero-Gallegos ${ }^{2}$, J.A. Sánchez-Fuentes ${ }^{2}$ y M. Martínez-Molina ${ }^{2}$ \\ 1 Facultad de Ciencias del Deporte, Universidad de Murcia, España,; 2 Centro del Profesorado Cuevas-Olula, Consejería de Educación, Cultura y Deporte, \\ Junta de Andalucia, España.
}

\begin{abstract}
Resumen: El objetivo de este trabajo fue conocer como el apoyo a la autonomía percibida, motivación y satisfacción hacia la Educación Física, predicen la importancia y utilidad de dicha asignatura. La muestra fue de 758 estudiantes (347 chicos y 411 chicas) de secundaria de la Región de Murcia, de entre 13 y 18 ańos. Se utilizó un cuestionario compuesto el Cuestionario de Clima de Aprendizaje, Escala de Motivación Deportiva, Escala de Satisfacción hacia la Educación Física y Escala de Importancia y Utilidad de la Educación Física. Se realizaron análisis descriptivos, correlacionales y ecuaciones estructurales. Los resultados indican una gran motivación y satisfacción hacia la asignatura, siendo siempre mayor en los chicos los resultados de todas las variables analizadas. El estudio demuestra la influencia del apoyo a la autonomía en la importancia de la Educación Física a través de la motivación intrínseca y la satisfacción del alumnado.

Palabras clave: apoyo a la autonomía; motivación; satisfacción; Educación Física

Abstract: The aim of this study was to understand how the perceived autonomy support, motivation and satisfaction towards Physical Education (PE), can predict the importance and usefulness of this subject. The sample consisted of 758 students ( 347 boys and 411 girls) of high school in the Region of Murcia, between 13 and 18 years old. The questionnaire was composed of Learning Climate Questionnaire, Sport Motivation Scale, Satisfaction Scale and Scale of Importance toward EF and EF Utility. It was
\end{abstract}

performed descriptive and correlational analyzes and structural equations. The results indicate a strong motivation and satisfaction towards the subject, being always greater in boys the results of all the variables analyzed. The study demonstrates the influence of autonomy support in the importance of EF through intrinsic motivation and students satisfaction.

Key words: autonomy support; motivation; satisfaction; Physical Education.

Resumo: O objectivo deste trabalho foi conhecer como o apoio à autonomia percebida, motivação e satisfação face à Educação Física, predizem a importância e utilidade dessa disciplina. A amostra foi constituída por 758 estudantes (347 rapazes e 411 raparigas) do ensino secundário da Região de Múrcia, com idades compreendidas entre os 13 e os 18 anos. Foi utilizado um questionário composto, o Questionário de Clima de Aprendizagem, Escala de Motivação Desportiva, Escala de Satisfação face à Educação Física e Escala de Importância e Utilidade da Educação Física. Realizaram-se análises descritivas, correlaçóes e equações estruturais. Os resultados indicam uma elevada motivação e satisfação face à disciplina, sendo sempre mais elevada nos rapazes em todas as variáveis analisadas. O estudo demonstra a influência do apoio à autonomia na importância da Educação Física através da motivação intrínseca e da satisfação dos alunos.

Palavras-chave: apoio à autonomia; motivação; satisfação; Educação Física.

\section{Introducción}

En los últimos años se ha producido un creciente interés por el estudio de la importancia y utilidad que el alumnado concede a la Educación Física (EF) (Baena-Extremera, GraneroGallegos, Pérez-Quero, Bracho-Amador, y Sánchez-Fuentes, 2013; Granero-Gallegos, Baena-Extremera, Pérez-Quero, Ortiz-Camacho, y Bracho-Amador, 2012; Moreno, González-Cutre, y Ruiz, 2009a; Moreno-Murcia, Zomeño, Marín, Ruiz, y Cervelló, 2013). El interés se ve incrementado debido a varios factores: por un lado, a la misión del área de EF dentro del currículum escolar en la consecución de hábitos de cuidado y salud corporales, donde hace una década autores como Kilpatrick, Hebert y Jacobsen (2002) anunciaban la relación entre la importancia concedida a la EF y la generación de

Dirección para correspondencia [Correspodence address]: Antonio Granero-Gallegos. Centro del Profesorado Cuevas-Olula, Carretera de Águilas s/n, Cuevas del Almanzora (Almería) 04610.Tlf: 0034637738201 E-mail: agranerog@hotmail.com hábitos físicos y deportivos en tiempo libre; y, por otro lado, a la incorporación de la EF y la práctica del deporte para favorecer el desarrollo personal y social, tal como queda recogido en el art. 23, objetivo K de la etapa de Educación Secundaria (Ley 2/2006 de Educación).

En este sentido, un aspecto relevante y que contribuye al estudio de esta variable, es que desde la normativa educativa se persigue que durante la escolaridad obligatoria y postobligatoria la EF contribuya a que el alumnado adquiera hábitos saludables de práctica física y deportiva en su tiempo libre y duraderos en el tiempo. Estos hábitos son determinantes en la formación integral del alumnado para su incorporación en una sociedad en la que es necesario estudiar, aprender, trabajar y disponer de hábitos de ocio y tiempo libre dentro de su rutina diaria.

Algunos estudios recientes, como los de Granero-Gallegos et al. (2012), Moreno, Zomeńo, Marín, Cervelló y Ruiz (2009b) y Moreno, Cervelló y González-Cutre (2007), encon- 
traron que los estudiantes que presentaban una alta importancia y utilidad a la EF eran los que mayor actividad física extraescolar practicaban. Pero la realidad, como ponen de manifiesto algunas investigaciones, es que entre los 12 y 18 años de edad es cuando se produce entre los adolescentes el mayor abandono en la práctica de actividad física (Caspersen, Pereira, y Curran, 2000, Gómez-López, Granero-Gallegos, y BaenaExtremera, 2010) debido, fundamentalmente, a la falta de motivación. Por ello, el estudio de los procesos motivacionales en el alumnado ha sido punto de mira de diversas investigaciones en los últimos años (Moreno-Murcia et al., 2013; entre otros), con el objetivo de encontrar soluciones a este problema.

Una de las teorías más utilizada para estudiar este fenómeno es la Teoría de la Autodeterminación, que analiza el grado en que las conductas humanas son volitivas o autodeterminadas; es decir, el grado en que las personas realizan sus acciones al nivel más alto de reflexión y se comprometen en las acciones con un sentido de elección (Deci y Ryan, 1985). Siguiendo una de las mini-teorías que componen la Autodeterminación, ésta se entiende como un continuo en el que se establecen diferentes niveles: de mayor a menor grado de autodeterminación, la conducta puede estar intrínsecamente motivada, extrínsecamente motivada o amotivada. El mayor grado de autodeterminación lo posee aquella persona que se encuentra intrínsecamente motivada, lo que conlleva un compromiso con la práctica deportiva, gracias al placer y al disfrute que obtiene con la misma, convirtiéndose ésta en un fin en sí misma (Deci y Ryan, 1985, 2000).

En el caso de la EF, la motivación es un factor clave que puede influir en los resultados del rendimiento académico, pues los altos logros de aprendizaje se atribuyen, a menudo, a la alta motivación de los estudiantes y a ambientes que favorecen la motivación (Moreno-Murcia, Sicilia, Cervelló, Huéscar, y Dumitru, 2011). Asimismo los perfiles más autodeterminados son los que más se relacionan con la importancia y utilidad de la EF (Borges, Belando, y Moreno-Murcia, 2014) y con la satisfacción, así como con el mayor número de horas de práctica física y deportiva en tiempo libre del alumnado (Granero-Gallegos et al., 2012; Moreno-Murcia et al., 2013), aspectos de interés para esta investigación.

La satisfacción del estudiante hacia la EF también es una variable importante a tener en cuenta (Baena-Extremera, Granero-Gallegos, Bracho-Amador, y Pérez-Quero, 2012). Ntounamis (2005) concluyó que cuando un alumno se encuentra satisfecho y se divierte tiende a estar intrínsecamente motivado, lo que supone una mayor participación en clases de EF e, incluso, una mayor práctica de actividad física en su tiempo libre. En esta línea, Granero-Gallegos et al. (2012) encontraron que los estudiantes de EF con un perfil de satisfacción/diversión, eran también alumnos con un perfil autodeterminado hacia las clases, que valoraban el esfuerzo y el trabajo duro por mejorar, dándole una gran importancia a la asignatura. Por tanto, es fundamental conocer al alumno en estos aspectos, pues de su satisfacción a esta asignatura y a la actividad física podrán inculcarse hábitos de ejercicio físico e, incluso, se podrá mejorar el rendimiento escolar, como ya demuestran algunos estudios (Ardoy, et al, 2013; Booth et al., 2014).

Por otro lado, algunos trabajos han comprobado que los climas de aprendizaje en los que se potencia el apoyo a la autonomía por parte del profesor hacia los estudiantes mejora la motivación de estos últimos (How, Whipp, Dimmock, y Jackson, 2013; Matos, 2009). Concretamente, El apoyo a la autonomía supone una variable importante y a tener en cuenta en la enseñanza; sobre todo cuando las leyes educativas españolas incluyen la autonomía e iniciativa personal entre las competencias básicas que el alumnado debe adquirir durante su etapa escolar. Si se pretende formar a estudiantes autónomos y con capacidad de decisión e iniciativa, el docente juega un papel fundamental pues, como demostraron Hagger, Chatzisarantis, Barkoukis, Wang y Baranowski (2005), la percepción de apoyo a la autonomía que tiene el alumno por parte del profesor en el contexto de la EF influye en el comportamiento posterior que el estudiante tiene en su tiempo libre.

Teniendo en cuenta lo expuesto, se aprecia cierta relación entre la importancia y utilidad de la EF con la consecución de hábitos de salud y de práctica física. Pero además, se aprecia por los trabajos citados, la relación existente entre estos hábitos y la motivación, la satisfacción y la percepción de apoyo a la autonomía en EF. Lo que hasta día de hoy no se conoce, es como conseguir a partir del conjunto de estas variables, que el alumno otorgue mayor importancia y utilidad a dicha asignatura, para poder responder al interés que muestra esta variable, como se menciona al inicio de la introducción. Por este motivo, se cree necesario acometer un trabajo que aborde conjuntamente el apoyo a la autonomía percibido, la motivación autodeterminada, satisfacción e importancia y utilidad concedida por el estudiante a la EF, para determinar un modelo predictivo entre las expuestas variables, así como observar las relaciones entre las mismas. A partir de los resultados de los trabajos anteriormente expuestos, se hipotetiza que el apoyo a la autonomía predecirá positivamente la motivación intrínseca, ésta la satisfacción/diversión en EF y, finalmente, ésta última la importancia de la EF. Se pretende así obtener información que permita mejorar los planteamientos e intervenciones educativas por parte de los docentes de EF y conocer las diferencias en estas variables entre sexos.

\section{Método}

\section{Participantes}

La selección de la muestra fue de tipo no probabilístico y por conveniencia, según los sujetos a los que se pudo acceder. Par- 
ticiparon un total de 758 alumnos (347 hombres=45.8\%; 411 mujeres $=54.2 \%$ ) de educación secundaria de centros públicos de la Región de Murcia. El rango de edad estuvo comprendido entre 13 y 18 ańos $(M=15.22 ; D T=1.27)$, siendo la edad media de los chicos $15.2(D T=1.29)$ y la de las chicas 15.18 $(D T=1.26)$. La distribución por cursos fue la siguiente: 343 (45.3\%) estudian 2o de ESO; 152 (20.1\%), 3º de ESO; 206 (27.2\%), 40 de ESO; y 57 (7.5\%), $1^{\circ}$ de bachillerato.

\section{Instrumentos}

Learning Climate Quetionarire (LCQ). Se utilizó la versión española adaptada a EF (LCQ-EF) (Granero-Gallegos, Baena-Extremera, Sánchez-Fuentes, y Martínez-Molina, in press) del original de Wiliamns y Deci (1996), basada en el Health-Care Climate Questionnaire (Williams, Grow, Freedman, Ryan, y Deci, 1996). Consta de 14 ítems para medir el apoyo a la autonomía por parte del profesor, mediante una dimensión que se denomina: apoyo a la autonomía. En las instrucciones se pide a los participantes que indiquen el grado de acuerdo con los ítems, recogiéndose las respuestas en una escala de ítems politómicos de 7 puntos que oscila desde $M u y$ en desacuerdo (1) a Muy de acuerdo (7). Esta escala mostró una alta consistencia interna, alfa de Cronbach $(\alpha)=.95$.

Sport Motivation Scale (SMS). Se utilizó la versión validada al español por Núñez, Martín-Albo, Navarro, y González (2006) y adaptada a la EF. La escala original se denominó Échelle de Motivationdans les Sports (EMS; Brière; Vallerand, Blais, y Pelletier, 1995) y fue traducida al inglés por Pelletier et al. (1995) pasando a denominarse Sport Motivation Scale $(S M S)$, y se obtuvieron similares propiedades psicométricas que en la versión francesa. Consta de 28 ítems que miden los diferentes tipos de motivación establecidos por la teoría de la Autodeterminación (Deci y Ryan, 1985), que sugiere la explicación multidimensional de la motivación: amotivation (AMO) (4 ítems), motivación extrínseca (ME) (12 ítems; identificada, introyectada y de regulación externa) y motivación intrínseca (MI) (12 ítems; estimulación, logro y conocimiento). Las respuestas fueran recogidas en una escala de ítems politómicos con un rango de puntuación entre 1 (totalmente en desacuerdo) y 7 (totalmente de acuerdo). La consistencia interna hallada en el presente estudio fue: $M I$, alfa de Cronbach $(\alpha)=.93 ; M E, \alpha=.91 ;$ y $A M O, \alpha=.75$.

Sport Satisfaction Instrument (SSI). Se utilizó la versión española del Sport adaptada a EF (SSI-EF) (Baena-Extremera et al., 2012) del original Sport Satisfaction Instrument (Balaguer, Atienza, Castillo, Moreno, y Duda, 1997; Duda y Nicholls, 1992). El SSI-EF consta de 8 ítems para medir la satisfacción intrínseca en una actividad deportiva mediante dos subescalas que miden satisfacción/diversión (SAT/D) (5 ítems) y aburrimiento (ABU) (3 ítems) en la práctica deportiva. En las instrucciones se pide a los participantes que indiquen su grado de acuerdo con los ítems que reflejan criterios de diversión o aburrimiento, recogiéndose las respuestas en una escala de ítems politómicos de 5 puntos que oscila desde muy en desacuerdo (1) a muy de acuerdo (5). La consistencia interna de la subescala satisfacción/diversión fue de $\alpha=.77$ y la de aburrimiento, $\alpha=.71$.

Importancia y utilidad de la Educación Física (IEF). Se midió la importancia y utilidad concedida por el alumnado a la EF (Moreno et al., 2009a) a través de 3 ítems. Los alumnos debían responder en una escala de ítems politómicos con un rango de puntuación entre 1 (totalmente en desacuerdo) y 4 (totalmente de acuerdo). Estudios previos demostraron la validez interna y su fiabilidad en el ámbito de la EF y en estudiantes adolescentes: $\alpha=.75$ (Moreno et al., 2009a); $\alpha=.76$ (Granero-Gallegos et al., 2012). En este estudio la fiabilidad obtenida fue .76 .

\section{Procedimiento}

Fue necesario pedir permiso previo a los órganos competentes, para poder llevar a cabo esta investigación. Posteriormente, se informó en detalle a padres/tutores y adolescentes acerca del protocolo y objeto del estudio, de la voluntariedad para participar y de la confidencialidad de las respuestas y datos, siendo un requisito indispensable la firma del consentimiento informado por parte de ambos. Los instrumentos para medir las diferentes variables se administraron en el aula por los propios investigadores y sin la presencia del docente, dejando un tiempo de entre 30 y 45 minutos. Se les informó de que no había respuestas correctas o incorrectas, pidiéndoles al comienzo de éste que contestaran con la máxima sinceridad y honestidad. Las dudas surgidas en los adolescentes durante el trabajo de campo fueron resueltas en ese momento por el equipo de investigadores.

\section{Análisis estadísticos}

Los estadísticos descriptivos, correlaciones (coeficiente de Pearson) entre las dimensiones analizadas del LCQ-EF, SMS, SSI-EF e IEF, consistencia interna (alfa de Cronbach) y diferencias según la variable sexo, se realizaron con el SPSS 17.0. Para estudiar la predicción de la importancia y utilidad concedida por el alumnado a la EF según el apoyo a la autonomía, motivación y satisfacción en EF se utilizó un Modelo de Ecuaciones Estructurales (SEM) realizado con LISREL 8.80.

\section{Resultados}

\section{Análisis descriptivo y correlaciones bivariadas}

En la Tabla 1 se muestran los estadísticos descriptivos de las variables utilizadas así como su correlación. En relación al 
análisis de correlación destaca la alta y positiva significatividad entre la motivación intrínseca y la motivación extrínseca, así como la correlación entre el apoyo a la autonomía y la motivación intrínseca y motivación extrínseca. Resalta también la positiva y significativa relación entre la motivación intrínseca y la satisfacción/diversión. Se ha de destacar las significativas y negativas relaciones entre el aburrimiento y el resto de factores, a excepción de la amotivación con la que correlaciona positivamente.

Tabla 1. Media $(M)$, desviación típica $(D T)$ y correlaciones entre las dimensiones analizadas.

\begin{tabular}{lccccccccc}
\hline & $M$ & $D T$ & 1 & 2 & 3 & 4 & 5 & 6 & 7 \\
\hline 1. Apoyo a la autonomía & 4.74 & 1.57 & - & $.66^{* *}$ & $.61^{* *}$ & $.15^{* *}$ & $.56^{* *}$ & $-.34^{* *}$ & $.19^{* *}$ \\
2. Motivación intrínseca & 4.92 & 1.33 & - & - & $.88^{* *}$ & $.17^{* *}$ & $.64^{* *}$ & $-.33^{* *}$ & $.19^{* *}$ \\
3. Motivación extrínseca & 4.85 & 1.25 & - & - & - & $.29^{* *}$ & $.59^{* *}$ & $-.27^{* *}$ & $.20^{* *}$ \\
4. Amotivación & 3.72 & 1.57 & - & - & - & - & .04 & $.20^{* *}$ & $.14^{* *}$ \\
5. Satisfacción/Diversión & 3.92 & 1.20 & - & - & - & - & - & $-.51^{* *}$ & $.21^{* *}$ \\
6. Aburrimiento & 2.18 & 1.01 & - & - & - & - & - & - & -.06 \\
7. Importancia de la EF & 2.79 & 1.14 & - & - & - & - & & - & - \\
\hline
\end{tabular}

**La correlación es significativa al nivel .01 (bilateral)

\section{Diferencias según las variables sexo y edad}

Para analizar la interacción del sexo y la edad de los estudiantes se realizó un análisis de varianza multivariado (MANOVA) 2 (sexo) x 3 (edad). El sexo y la edad fueron considerados como variables independientes, mientras que las subescalas del LCQ-EF, SMS, SSI-EF e IEF actuaron como variables dependientes. No se hallaron diferencias significativas en el contraste multivariado y efectos de interacción entre las dos variables independientes (Lambda de Wilks=.96, $\mathrm{F}_{(14}$, $\left.{ }_{1488}=2.50, \mathrm{p}=.006\right)$. Las pruebas de efectos intersujetos sí mostraron diferencias significativas en función de la variable sexo (Lambda de Wilks=.961; $\left.\mathrm{F}_{(7,743)}=4.27 ; \mathrm{p}<.001\right)$, pero no en relación a la edad del alumnado (Lambda de Wilks=.980; $\mathrm{F}_{(14}$ $\left.{ }_{1486)}=1.10 ; \mathrm{p}=.354\right)$. En relación al sexo, como se puede observar en la Tabla 2, se encontraron diferencias en la motivación intrínseca, motivación extrínseca, amotivación, satisfacción, e importancia y utilidad de la EF, siendo mayor las medias calculadas en los chicos que en las chicas.

Tabla 2. Análisis multivariante según sexo y edad.

\begin{tabular}{lcccccccccccccccc}
\hline & \multicolumn{1}{c}{ Sexo } & \multicolumn{1}{c}{$\begin{array}{c}\text { Edad } \\
(n=347)\end{array}$} & $\begin{array}{c}\text { Chicas } \\
(n=411)\end{array}$ & & \multicolumn{4}{c}{$\begin{array}{c}12-14 \text { años } \\
(n=374)\end{array}$} & $\begin{array}{c}15-16 \text { años } \\
(n=316)\end{array}$ & $\begin{array}{c}17-19 \text { años } \\
(n=65)\end{array}$ \\
\hline & $M$ & $D T$ & $M$ & $D T$ & $F$ & $p$ & $M$ & $D T$ & $M$ & $D T$ & $M$ & $D T$ & $F$ & $p$ \\
\hline Apoyo a la autonomía & 4.81 & 1.49 & 4.70 & 1.64 & .82 & .367 & 4.73 & 1.66 & 4.81 & 1.47 & 4.66 & 1.50 & .41 & .609 \\
\hline Motivación intrínseca & 5.01 & 1.29 & 4.82 & 1.39 & 7.13 & .008 & 4.90 & 1.41 & 4.99 & 1.29 & 4.98 & 1.31 & .59 & .557 \\
\hline Motivación extrínseca & 5.04 & 1.21 & 4.71 & 1.26 & 13.61 & .000 & 4.84 & 1.30 & 4.88 & 1.18 & 4.84 & 1.22 & .18 & .832 \\
\hline Amotivación & 3.89 & 1.58 & 3.60 & 1.54 & 6.48 & .011 & 3.78 & 1.60 & 3.74 & 1.53 & 3.38 & 1.61 & 1.82 & .163 \\
\hline Satisfacción/Diversión & 4.10 & 1.17 & 3.80 & 1.12 & 20.59 & .000 & 3.92 & 1.04 & 3.94 & 1.07 & 3.97 & 1.01 & .07 & .933 \\
\hline Aburrimiento & 2.20 & 1.05 & 2.16 & 1.01 & .37 & .543 & 2.26 & 1.14 & 2.12 & .94 & 2.33 & 1.05 & 1.02 & .361 \\
\hline Importancia de la EF & 2.93 & 1.08 & 2.68 & 1.17 & 22.31 & .000 & 2.80 & 1.06 & 2.75 & 1.18 & 2.92 & .94 & 1.65 & .194 \\
\hline Nota. pes significativo al nivel <.05 & & & & & & & & & & & & \\
\hline
\end{tabular}

\section{Modelo de Ecuaciones Estructurales}

Con el objeto de comprobar la relación predictiva entre las dimensiones estudiadas se siguió el método de dos pasos propuesto por Anderson y Gerbing (1988) (paso 1: modelo de medida; paso 2: modelo de ecuaciones estructurales). De esta forma, inicialmente se llevó a cabo un análisis factorial confirmatorio (AFC) de cada una de las escalas para valorar 
posteriormente el modelo de medida. Este análisis permitió confirmar la estructura factorial de las escalas empleadas en el estudio, así como evaluar su validez de constructo.

Con el objeto estudiar las propiedades psicométricas de la dimensionalización original propuesta teóricamente en cada una de las escalas, se aplicaron modelos de ecuaciones estructurales, evaluándose con AFC. Debido a la falta de normalidad de los datos, este análisis se realizó utilizando el método de estimación weighted least squares (WLS) para variables ordinales del programa LISREL 8.80 (Jöreskog y Sörbom, 1993). La matriz de correlaciones policóricas y la matriz de covarianzas asintóticas fueron utilizadas como input para el análisis de los datos. Se hipotetizó para cada escala un modelo de medida consistente en un modelo de factores que asumió la existencia de las variable latentes según los instrumentos originales descritos en el apartado correspondiente. En la escala de tres variables, atendiendo las sugerencias de Jöreskog y Sörbom (1993), para el AFC se fijó la varianza del error de una de las variables observadas.

Siguiendo las indicaciones de Bentler (2007), para llevar a cabo la evaluación de los modelos se calcularon varios índices de ajuste, usando una combinación de índices absolutos y relativos. Entre los absolutos, se utilizó el valor p, asociado con el estadístico chi cuadrado $\left(\chi^{2}\right)$, que prueba el modelo nulo frente al modelo hipotetizado (Barrett, 2007). La ratio entre $\chi^{2}$ y grados de libertad $(\mathrm{gl})\left(\chi^{2} / \mathrm{gl}\right)$ es un heurístico que se utiliza para reducir la sensibilidad del $\chi^{2}$ al tamaño de la muestra. Asimismo, se ha calculado el GFI (índice de bondad de ajuste), que indica la cantidad relativa de varianza y covarianza reproducida por el modelo específico, comparado con el modelo saturado. Entre los índices relativos se ha utilizado el NFI (índice de ajuste normalizado), el NNFI (índice de ajuste no normativo) y CFI (índice de ajuste comparativo). Además, autores como Kline (2005), recomiendan en estos modelos la utilización del error de aproximación cuadrático medio (RMSEA). Finalmente, los parámetros estimados se consideraron significativos cuando el valor asociado al valor $\mathrm{t}$ era superior a $1.96(p<.05)$.

En relación al LCQ-EF, los índices de ajuste obtenidos en el AFC fueron: $\chi^{2}=201.75, \mathrm{gl}=77, p<.000, \chi^{2} / \mathrm{gl}=2.62$, $\mathrm{GFI}=.99, \mathrm{NFI}=.98, \mathrm{NNFI}=.98, \mathrm{CFI}=.99$, RMSEA=.04. En el SMS, los índices de ajuste obtenidos fueron: $\chi^{2}=1047.22$, $\mathrm{gl}=347, p<.000, \chi^{2} / \mathrm{gl}=3.02, \mathrm{GFI}=.98, \mathrm{NFI}=.96, \mathrm{NNFI}=.97$, $\mathrm{CFI}=.98, \mathrm{RMSEA}=.05 \mathrm{El}$ SSI-EF presentó un aceptable ajuste: $\chi^{2}=49.86, \mathrm{gl}=19, p=.001, \chi^{2} / \mathrm{gl}=2.62, \mathrm{GFI}=.95, \mathrm{NFI}=.98$, $\mathrm{NNFI}=.99, \mathrm{CFI}=.99$, RMSEA=.05. El IEF presentó los siguientes índices de ajuste: $\chi^{2}=2.94, \mathrm{gl}=1, p<.09, \chi^{2} / \mathrm{gl}=2.94$, $\mathrm{GFI}=.99, \mathrm{NFI}=.99$, NNFI=.97, $\mathrm{CFI}=.99$, $\mathrm{RMSEA}=.01$.

Estos datos se ajustan a los parámetros establecidos, por lo que se puede aceptar como bueno el modelo propuesto ( $\mathrm{Hu}$ y
Bentler, 1999). De igual modo, la contribución de cada uno de los factores a la predicción de otras variables se examinó a través de los pesos de regresión estandarizados. El t-value asociado a cada peso se tomó como una medida de la contribución, de modo que valores $>1.96$ se consideran como significativos.

En el AFC de las escalas con naturaleza ordinal de la matriz de correlaciones de datos se considera importante ofrecer los resultados de fiabilidad compuesta y varianza media extraida (AVE - Average Variance Extracted) para cada una de las dimensiones críticas. Según Hair, Black, Babin y Anderson (2009), la fiabilidad compuesta debe tener un valor mínimo de .70 y la $A V E$ de .50 . En la Tabla 3 se pueden observar los positivos datos de consistencia interna y validez de la cada una de las dimensiones.

Tabla 3. Consistencia interna y validez de las dimensiones estudiadas.

\begin{tabular}{lccc}
\hline Dimensiones & Fiabilidad compuesta & $A V E$ & $\alpha$ \\
\hline Apoyo a la autonomía & .99 & .86 & .96 \\
\hline Motivación intrínseca & .99 & .92 & .91 \\
\hline Motivación extrínseca & .99 & .88 & .91 \\
\hline Aamotivación & .85 & .58 & .75 \\
\hline Satisfacción/Diversión & .90 & .64 & .77 \\
\hline Aburrimiento & .78 & .55 & .71 \\
\hline Importancia de la EF & .85 & .65 & .76 \\
\hline
\end{tabular}

Finalmente, para estudiar las relaciones e interacciones existentes entre las variables pertenecientes al modelo que se plantea se ha utilizado el Modelo de Ecuaciones Estructurales. Atendiendo a las recomendaciones de autores como Levy y Hancock (2007), de formular y analizar varios modelos, se llevaron a cabo diversos análisis. Para ello se utilizaron los índices de modificación que, según Cea (2002), informan sobre aquellas relaciones que pueden ser suprimidas o incorporadas para que el modelo mejore su ajuste, debiendo cumplir, para ser aceptados éstos índices, dos condiciones fundamentales: mejorar de forma sensible el nivel de ajuste del modelo y poder justificar teóricamente los cambios propuestos. De esta forma, se obtuvo como resultado final el modelo que mejor se ajusta según las variables estudiadas, tal como se representa en la Figura 1. Los resultados obtenidos de este modelo fueron: $\chi 2=3324.42, \mathrm{gl}=1320, p<.000, \chi^{2} / \mathrm{gl}=2.51, \mathrm{GFI}=.96$, $\mathrm{NFI}=.94, \mathrm{NNFI}=.96, \mathrm{CFI}=.99$, RMSEA=.03. Los datos, según las indicaciones anteriores, mostraron que el modelo de medición era adecuado. Los datos ofrecidos en la Figura 1, muestran 7 variables latentes con un total de 53 variables observadas. 
Figura 1: Análisis confirmatorio compuesto por 7 factores hipotetizados. Los círculos representan los constructos latentes y los cuadrados representan las variables medidas. Todos los parámetros son estandarizados y significativos en $p<.05$. (MI: motivación intrínseca; ME: motivación extrínseca; AMO: amotivación; SAT/D: satisfacción/diversión; ABU: aburrimiento; IEF: importancia y utilidad de la Educación Física)

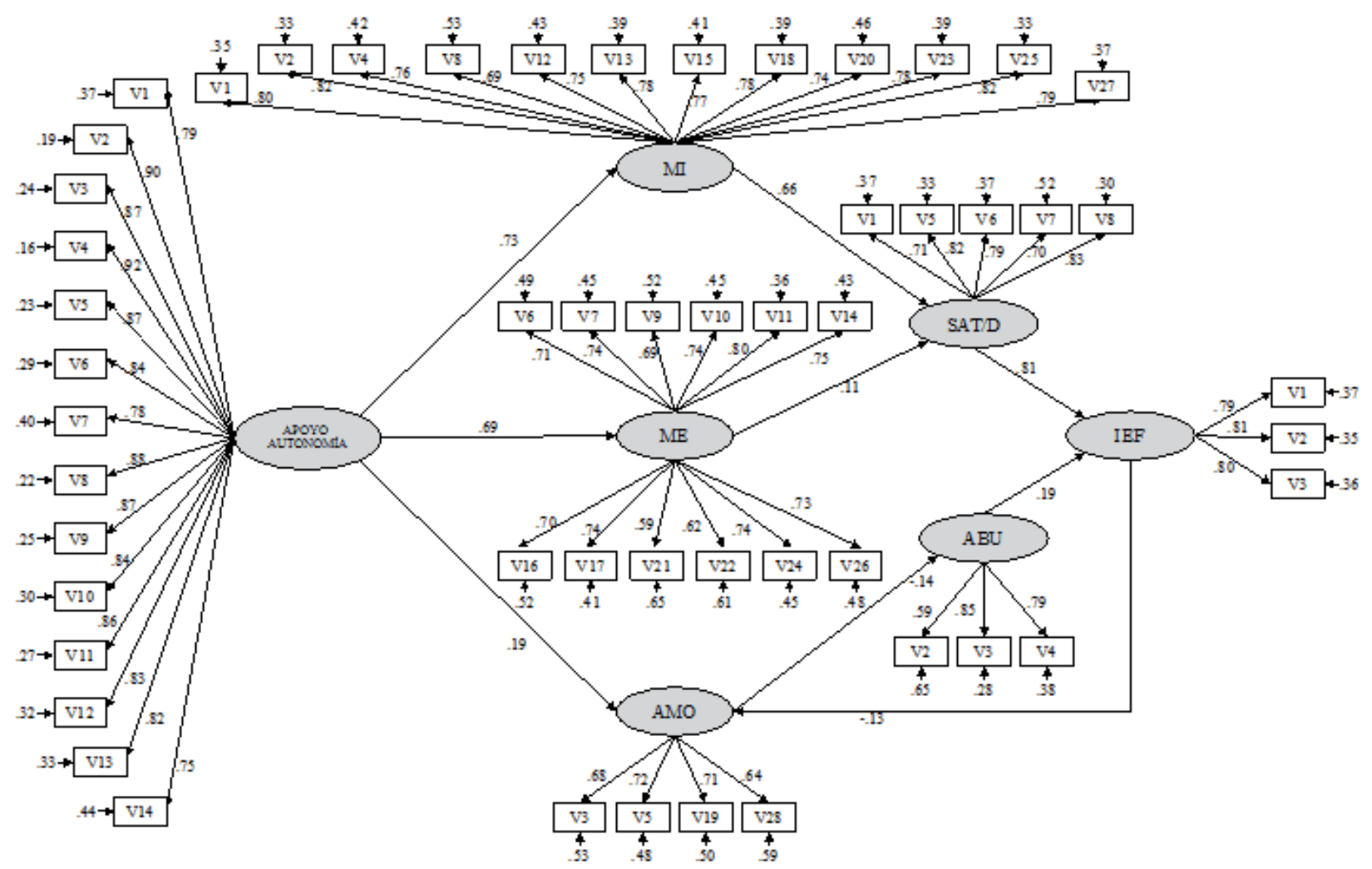

Los datos aportados corroboran un buen ajuste del modelo. De igual modo, la contribución de cada uno de los factores a la predicción de otras variables se examinó a través de los pesos de regresión estandarizados. En la Figura 1 se observa que el apoyo a la autonomía predice fundamentalmente la motivación intrínseca (.73) y la motivación extrínseca (.69). A continuación, es la motivación intrínseca la que predice fundamentalmente la satisfacción/diversión (.66), mientras que la motivación extrínseca presenta menos influencia (.11). Finalmente, la satisfacción/diversión establece una fuerte relación de predicción con la importancia y utilidad de la EF (.81). Por otro lado, los valores hallados en relación con la amotivación y el aburrimiento son bajos respecto a la predicción de la importancia de la EF.

\section{Discusión}

En este trabajo se ha abordado de forma conjunta el apoyo a la autonomía percibida por el alumno, la motivación, la satisfacción e importancia y utilidad de la EF, para determinar un modelo predictivo de las primeras variables sobre la última.
La importancia de este trabajo radica en que hasta ahora se ha venido estudiando de forma aislada la relación entre la importancia y utilidad de la EF con los hábitos de práctica física, pero no, como llegar a predecir esta variable a través de las variables utilizadas en un modelo de ecuaciones estructurales.

Entre los resultados hallados en este trabajo destaca, con una media más alta, la motivación intrínseca del alumnado de EF; estos datos se manifiestan en la línea de los hallazgos de otros autores en trabajos anteriores, como Granero-Gallegos et al. (2012) y Moreno-Murcia et al. (2013), lo que denota en estas investigaciones el interés del alumnado por esta asignatura, aspecto que los docentes deben aprovechar en sus clases y en su tarea docente. Es más, Lonsdale, Sabiston, Raedeke, Ha y Sum (2009), concluyen que el tipo de motivación que se asocia perfectamente con el compromiso con el área de la EF es la motivación intrínseca.

Es destacable también, como la satisfacción/diversión obtiene valores por encima del aburrimiento (corroborando las aportaciones de Baena-Extremera et al., 2012; Hernández, Velázquez, Martínez, Garoz, y López, 2008), y como la 
importancia y utilidad de la EF, obtiene valores medios. Sobre esto, Rodríguez, García-Cantó, Sánchez-López y LópezMińarro (2013) obtienen también que el 85\% del alumnado estudiado considera que la EF posee regular o baja utilidad, aspecto que creemos, el docente debe invertir de forma clamorosa.

Respecto al apoyo a la autonomía, resultó significativa la relación con la motivación intrínseca y la motivación extrínseca y es de destacar las relaciones negativas del aburrimiento con la mayoría de los factores, exceptuando la amotivación, lo que evidencia la validez empírica de estos instrumentos. En relación con estos resultados, trabajos ya realizados por otros autores han predicho que los climas de aprendizaje que apoyan a la autonomía de los estudiantes mejorarán su motivación intrínseca (Black y Deci, 2000; Deci, Schwartz, Scheinman, y Ryan, 1981), mientras que los climas en los que los estudiantes perciben un control de su comportamiento, disminuye su sentido de la voluntad, y se reduce la motivación natural. A esto, hay que sumarle que según Matos (2009), la motivación intrínseca es el mejor ejemplo del comportamiento autónomo y, por lo tanto, autodeterminado. Por otro lado, la motivación extrínseca es más controlada y por tanto, sería menos autónoma. Así pues, tiene coherencia los resultados mostrados en esta investigación con las aportaciones de estos autores. Esto supone que cuando el docente ofrece opciones de elección al alumno, éste se encuentra más motivado ya que puede optar a diversas posibilidades según sus intereses.

En el trabajo de Gillison, Osborn, Standage y Skevington (2009), los investigadores descubrieron diferencias entre la motivación de los chicos y las chicas. En esta investigación, podemos aportar que el tipo de motivación en cambio es similar (motivación intrínseca), aunque con valores bien distintos, siendo mayor en los varones. Corroborando estas aportaciones, en el trabajo llevado a cabo por Granero-Gallegos et al. (2012), Moreno, Rodríguez y Gutiérrez (1996), Moreno y Cervelló (2003), y Vlachopoulos, Karageorghis y Terry (2000), también se obtiene valores mayores de autodeterminación por parte de los chicos, aportando validez a estos hallazgos y mostrando una tendencia general entre los sexos, de los sujetos estudiados. Este resultado puede deberse en parte a la forma de actuar del docente, ya que por lo general, se cree que en las clases de EF se crea un clima más de rendimiento, lo que potencia, sobre todo en los chicos, que lleguen a estar más autodeterminados (Granero-Gallegos y Baena-Extremera, 2014). Por eso, en muchos trabajos se terminan concluyendo la necesidad de orientar las clases más hacia un clima tarea (Gutiérrez, Ruíz, y López, 2011), donde ahí, posiblemente las chicas aumentarán más su motivación.

$Y$ en relación a la satisfacción y diversión, Ntounamis (2005) llegó a la conclusión que cuando un alumno se divierte tiende a estar intrínsecamente motivado, lo cual se entiende como lógica esta relación.
En cambio, en relación a la amotivación, la mayoría de estudios afirman que son las chicas las que suelen presentar mayores valores de amotivación (Granero-Gallegos et al., 2012), datos que aquí no se pueden defender como se aprecia en la tabla 2, pero que pueden explicar la tasa de abandono o de no práctica física encontrada en otros trabajos por las féminas (Rodríguez et al., 2013). Además, la falta de motivación en las chicas puede deberse según (Amado et al., 2014) a razones socio-culturales, entre las que se podría encontrar la preferencia de ellas por otros contenidos menos trabajados o con menos peso en EF, como son las danzas (O'Neill, Pate y Liese, 2011).

Como se aprecia en los resultados, el apoyo a la autonomía predice principalmente la motivación intrínseca, extrínseca y, en último lugar, la amotivación. Así, el apoyo a la autonomía por parte del profesorado de EF se mostró como un predictor preferentemente de las formas más autodeterminadas de motivación entre los estudiantes adolescentes, coincidiendo con el modelo de Vallerand (1997) y en concordancia con los trabajos de Brickell, Chatzisarantis y Pretty (2006), Chatzisarantis, Hagger, Smith, y Sage (2006), Hagger et al. (2005, 2009), Lim y Wang, (2009) y Standage, Gillison, Ntoumanis y Treasure (2012).

La motivación autodeterminada predice la satisfacción/ diversión, mientras que la amotivación es predictora del aburrimiento. Finalmente, la importancia y utilidad de la EF es predicha, sobre todo, por la satisfacción/diversión en las clases de EF. En el trabajo llevado a cabo por Haerens, Kirk, Cardon, De Bourdeaudhuij y Vansteenkiste (2010) con 2600 estudiantes, se demostró que los estudiantes con un perfil motivacional más óptimo para la EF (es decir, las puntuaciones altas en motivación autónoma para esta asignatura) eran más propensos a practicar actividad física fuera de la escuela, incluso en la edad adulta temprana. En cambio, los estudiantes con un perfil menos autodeterminado hacia EF (alumnos amotivados) eran menos propensos a trasladar esa práctica fuera del entorno escolar. Estos resultados se encuentran en la línea con otros estudios que muestran que las formas autónomas de motivación en EF pueden tener una influencia positiva en los hábitos de actividad física durante el tiempo libre (Cox, Smith, y Williams, 2008; Granero-Gallegos et al., 2012). Esto significa que la transferencia del aprendizaje es más probable que ocurra entre los alumnos que se encuentran clases de EF por sí agradable y significativas, de manera que se sientan satisfechos y divertidos, mientras que los estudiantes que se sienten presionados a participar y aburridos en esta asignatura, no reportaría dicha transferencia.

\section{Conclusiones}

Como conclusión final, resaltar los valores de motivación intrínseca, satisfacción, e importancia y utilidad de la EF, ma- 
yores siempre en los varones. Además, para conseguir que los alumnos alcancen una importancia y utilidad de dicha asignatura, se debería partir en las clases potenciando el apoyo a la autonomía del alumno, para conseguir una mayor autodeterminación, y así, una mayor satisfacción/diversión. De esta forma, sólo y exclusivamente (como se refleja en la Figura 1) podríamos llegar a conseguir la variable final.

\section{Aplicaciones prácticas}

Los avances sobre la motivación en el campo de la educación han establecido ciertas pautas que sirven al profesorado, en muchas ocasiones, de guía para el funcionamiento como docente en las clases (García-Dantas, Caracuel-Tubío, y Peñaloza-Gómez, 2013). En el caso que nos ocupa, los resultados aquí encontrados son especialmente importantes para la EF en la promoción de un estilo de vida activo en los adolescentes (How et al., 2013), sobre todo, cuando ya otros investigadores han encontrado predicción entre la importancia de la EF y la intención de continuar haciendo ejercicio físico por el alumnado (Moreno-Murcia, Huéscar, y Cervelló, 2012). Por un lado se ha de tener en cuenta que, según exponen Hagger et al. (2005, 2009), el apoyo a la autonomía influye en la intención de práctica de ejercicio físico en tiempo libre; y, por otro lado, que la importancia concedida a la EF entre estudiantes adolescentes se relacionan con perfiles autodeterminados o de alta motivación, así como con un número mayor de horas de práctica deportiva en tiempo de ocio (Granero-Gallegos et al., 2012; Moreno-Murcia et al., 2013). Así pues, la asignatura de EF y el profesorado de la misma tienen un papel muy activo ante esta situación. Por ello, los datos del presente estudio son muy importantes de cara a la preparación del profesorado y la organización de la docencia a nivel metodológico, con vistas a conseguir estos resultados.

A partir de la relevancia que adquiere la importancia y utilidad que el alumnado concede a la EF, el profesorado tiene un serio desafío: convertir en interesantes aquellas actividades deseables por los alumnos pero no siempre intrínsecamente interesantes (Standage, Gillison, y Treasure, 2007). Para ello, autores como Moreno y Martínez (2006), ofrecen algunas pautas para mejorar la autodeterminación del alumno, a lo que habría que sumarle otras estrategias como dar al estudiante opciones de elección del tipo de tarea (Chatzisarantis, Hagger, Biddle, Smith, y Wang, 2003; Hernández, Vasconcelos-Raposo, Lázaro, y Dosil, 2004), ofrecerles la opción de elegir la dificultad de la tarea a realizar (How et al., 2013), o establecer grupos de aprendizaje entre iguales en el que los estudiantes desempeñen papeles diferentes (demostraciones, arbitrajes, etc.) (Lim y Wang, 2009). Para poder llevar a cabo esto, y siguiendo las manifestaciones expresadas por otros autores (Barkoukis y Hagger, 2013; Cheon y Moon, 2010; Cheon, Reeve, y Moon, 2012; Moreno, Vera, y Del Villar, 2010; Su y Reeve, 2011), sería necesario formar al profesorado en habilidades didácticas que les ayuden a diseñar y llevar a cabo sesiones para aumentar la autonomía de los alumnos.

\section{Limitaciones y perspectivas de futuro}

Una de las principales limitaciones de esta investigación, es el tipo de muestra, que no el tamaño. En futuros trabajos, se podría llevar a cabo comparaciones no sólo entre comunidades autónomas o ciudades, sino entre tipos de nacionalidades y tipo de centros (públicos y privados) con realidades sociales y culturales diversas. Esto ayudaría a conocer algunas de las peculiaridades socio-culturales de estos alumnos en EF, pues como expresan Taylor y Lonsdale (2010), estas diferencias pueden provocar patrones motivacionales diferentes en relación a las preferencias de estos alumnos con respecto a la actividad física y la EF.

Otra de las posibles limitaciones, es el tipo de diseño utilizado, de carácter transversal. Sería de gran interés para la comunidad científica llevar a cabo una investigación pre-post, de carácter más longitudinal, con grupo experimental, donde se puedan conocer los efectos sobre estas variables tras una intervención. Esto ayudaría, a diseñar de cara al futuro, programas de formación del profesorado para poder intervenir en clases de EF potenciando la autonomía, la motivación, la satisfacción y la importancia y utilidad de la EF.

\section{Referencias}

1. Amado, D., Del Villar, F., Leo, F. M., Sánchez-Oliva, D., SánchezMiguel, P. A. y García-Calvo, T. (2014). Effect of a Multi-Dimensional Intervention Programme on the Motivation of Physical Education Students. Plos One, 9(1), e85275. doi:10.1371/journal.pone.0085275.

2. Anderson, J. C. y Gerbin, D. W. (1988). Structural equation modeling in practice: a review y recommended two-step approach. Psychological Bulletin, 103, 411-423.

3. Ardoy, D., Fernández-Rodríguez, J. M., Jiménez-Pavón, D., Castillo, R., Ruiz, J. R. y Ortega, F. B. (2013). A Physical Education trial improves adolescents' cognitive performance and academic achievement: the EDUFIT study. Scandinavian Journal of Medicine y Science in Sports, 5 , 1-10. doi: $10.1111 / \mathrm{sms} .12093$.
4. Baena-Extremera, A., Granero-Gallegos, A, Bracho-Amador, C. y Pérez-Quero, F.J. (2012). Versión española del Sport Satisfaction Instrument (SSI) adaptado a la Educación Física. Revista de Psicodidáctica, 17(2), 377-396. doi:10.1387/RevPsicodidact. 4037.

5. Baena-Extremera, A., Granero-Gallegos, A. Pérez-Quero, F. J., BrachoAmador, C. y Sánchez Fuentes, J. A. (2013). Prediction of perceived importance of physical education in Spain. South African Journal for Research in Sport, Physical Education and Recreation, 35(2), 1-13.

6. Balaguer, I., Atienza, F. L., Castillo, I., Moreno, Y. y Duda, J. L. (1997). Factorial structure of measures of satisfaction/interest in sport and classroom in the case of Spanish adolescents. Abstracts of $4^{\text {th }}$ European Conference of Psychological Assessment (p. 76). Lisbon: Portugal. 
7. Barkoukis, V. y Hagger, M. S. (2013). The trans-contextual model: perceived learning and performance motivational climates as analogues of perceived autonomy support. European Journal of Psychology of Education, 28(2), 353-372. doi: 10.1007/s10212-012-0118-5

8. Barrett, P. (2007). Structural equation modelling: Adjudging model fit. Personality and Individual Differences 42, 815-824. doi: 10.1016/j. paid.2006.09.018

9. Bentler, P. M. (2007). On tests and indices for evaluating structural models. Personality and Individual Differences, 42, 825-829.

10. Black, A. E. y Deci, E. L. (2000). The effects of instructors' autonomy support and students' autonomous motivation on learning organic chemistry: A self-determination theory perspective. Science Education, 84, 740-756. doi: 10.1002/1098-237X(200011)84:6<740::AIDSCE4>3.0.CO;2-3.

11. Borges, F., Belando, N. y Moreno-Murcia, N. (2014). Percepción de igualdad de trato e importancia de la educación física de alumnas adolescentes. Revista de Psicodidáctica, 19(1), 173-189. doi: 10.1387/RevPsicodidact.8007.

12. Booth, J. N., Leary, S. D., Joinson, C., l., Ness, A. R., Tomporowski, P. D., Boyle, J. M. y Reilly, J. J. (2014). Associations between objectively measured physical activity and academic attainment in adolescents from a UK cohort. British Journal of Sports Medicine, 48, 265-270. doi:10.1136/bjsports-2013-092334.

13. Brickell, T. A., Chatzisarantis, N. L. D. y Pretty, G. M. (2006). Autonomy and control: augmenting the validity of the theory of planned behaviour in predicting exercise. Journal of Health Psychology, 11, 51-63. doi: $10.1177 / 1359105306058847$.

14. Brière, N., Vallerand, R., Blais, N. y Pelletier, L. (1995). Développement et validation d'une mesure de motivation intrinsèque, extrinsèque et d'amotivation en contexte sportif :l'Échelle de motivation dans les sports (ÉMS). International Journal of Sport Psychology, 26, 465-489.

15. Caspersen, C. J., Pereira, M. A. y Curran, K. M. (2000). Changes in physical activity patterns in the United States, by sex and cross-sectional age. Medicine and Science in Sport and Exercise, 3285, 1601-1609.

16. Cea, M. A. (2002). Análisis multivariable. Teoría y práctica en la investigación social. Madrid: Síntesis.

17. Chatzisarantis, N. L. D., Hagger, M. S., Biddle, S. J. H., Smith, B. y Wang, J. C. K. (2003). A meta-analysis of perceived locus of causality in exercise, sport, and physical education contexts. Journal of Sport and Exercise Psychology, 25, 284-306.

18. Chatzisarantis, N. L. D., Hagger, M. S., Smith, B. y Sage, L. D. (2006). The influence of intrinsic motivation on execution of social behaviour within the theory of planned behaviour. European Journal of Social Psychology, 36, 229-237. doi: 10.1002/ejsp.299.

19. Cheon, S. H. y Moon, I. S. (2010). Implementing an autonomy-supportive fitness program to facilitate students' autonomy and engagement. Korean Journal of Sport Psychology, 21, 175-195.

20. Cheon, S. H., Reeve, J. y Moon, I. S. (2012). Experimentally based, longitudinally designed, teacher-focused intervention to help physical education teachers be more autonomy supportive toward their students. Journal of Sport and Exercise Psychology, 34, 365-396.

21. Cox, A. E., Smith, A. L. y Williams, L. (2008). Change in physical education motivation and physical activity behavior during middle school. Journal of adolescent health, 43, 506-513. doi: 10.1016/j.jadohealth.2008.04.020.

22. Deci, E. L. y Ryan, R. M. (1985). Intrinsic motivation and self-determination in human behavior. New York: Plenum.

23. Deci, E. L. y Ryan, R. M. (2000). The "what" and "why" of goal pursuits: Human needs and the self-determination of behavior. Psychological Inquiry, 11, 227-268. doi: 10.1207/S15327965PLI1104_01.

24. Deci, E. L., Schwartz, A. J., Sheinman, L. y Ryan, R. M. (1981). An instrument to assess adults' orientations toward control versus autonomy with children: Reflections on intrinsic motivation and perceived competence. Journal of Educational Psychology, 73, 642-650.

25. Duda, J. L. y Nicholls, J. G. (1992). Dimensions of achievement motivation in schoolwork and sport. Journal of Educational Psychology, 84(3), 290-299. doi: 10.1037/0022-0663.84.3.290.

26. García-Dantas, A., Caracuel-Tubío, J. C. y Peñaloza-Gómez, R. (2013). Intervención formativa con el profesorado de danza e influencia motivacional en su alumnado. Cuadernos de Psicología del Deporte, 13(2), 9-20.

27. Gillison, F., Osborn, M., Standage, M. y Skevington, S. (2009). Exploring the experience of introjected regulation for exercise across gender in adolescence. Psychology of Sport and Exercise, 10, 309-319. doi:10.1016/j. psychsport.2008.10.004.

28. Granero-Gallegos, A. y Baena-Extremera, A. (2014). Predicción de la motivación autodeterminada según las orientaciones de meta y el clima motivacional en Educación Física. Retos. Nuevas tendencias en Educación Física, Deporte y Recreación, 25, 23-27.

29. Granero-Gallegos, A., Baena-Extremera, A., Pérez-Quero, F. J., OrtizCamacho, M. M. y Bracho-Amador, C. (2012). Analysis of motivational profiles of satisfaction and importance of physical education in high school adolescents. Journal of Sports Science and Medicine, 11, 614-623.

30. Granero-Gallegos, A., Baena-Extremera, A., Sánchez-Fuentes, J. A. y Martínez-Molina, M. (in press). Validación española del "Learning Climate Questionaire” adaptado a la Educación Física. Psicologia: Reflexão e Crítica, 27(4).

31. Gómez-López, M., Granero-Gallegos, A. y Baena-Extremera, A. (2010) Perceived barriers by university students in the practice of physical activities. Journal of Sports Science and Medicine, 9, 374-381.

32. Gutiérrez, M., Ruiz, L. M. y López, E. (2011). Clima motivacional en Educación Física: concordancia entre las percepciones de los alumnos y las de sus profesores. Revista de Psicología del Deporte, 20(2), 321-335.

33. Haerens, L., Kirk, D., Cardon, G., De Bourdeaudhuij, I. y Vansteenkiste, M. (2010). The quality and quantity of motivation for secondary school physical education and its relationship to the adoption of a physically active lifestyle among university students. European Physical Education Review 16(2), 117-139. doi: 0.1177/1356336X10381304.

34. Hagger, M. S., Chatzisarantis, N., Barkoukis, V.,Wang, C. K. J. y Baranowski, J. (2005). Perceived autonomy support in physical education and leisure-time physical activity: a cross-cultural evaluation of the trans-contextual model. Journal of Educational Psychology, 97, 376-390. doi: 10.1037/0022-0663.97.3.376.

35. Hagger, M. S., Chatzisarantis, N. L. D., Hein, V., Pihu, M., Soos, I., Karsai, I., Lintunen, T. y Leemans, S. (2009). Teacher, peer, and parent autonomy support in physical education and leisure-time physical activity: A trans-contextual model of motivation in four nations. Psychology and Health, 24, 689-711. doi: 10.1080/08870440801956192.

36. Hair, J. F., Black, W. C., Babin, B. J. y Anderson, R. E. (2009). Multivariate Data Analysis (7th ed.). New York: Pearson Prentice Hall.

37. Hernández, H., Vasconcelos-Raposo, J., Lázaro, J. P. y Dosil. J. (2004). Validación y aplicación de modelos teóricos motivacionales en el contexto de la Educación Física. Cuadernos de Psicología del Deporte, 4(1-2), 67-89.

38. Hernández, J. L., Velázquez, R., Martínez, M. E., Garoz, I. y López, A. (2008). Frecuencia de actividad física en niños y adolescentes: relación con su percepción de autoeficacia motriz, la práctica de su entorno social y su satisfacción con la Educación Física. Infancia y Aprendizaje, 31(1), 79-92.

39. How, Y. M., Whipp, P. R., Dimmock, J. A. y Jackson, B. (2013). The Effects of Choice on Autonomous Motivation, Perceived Autonomy Support, and Physical Activity Levels in High School Physical Education. Journal of Teaching in Physical Education, 32, 131-148.

40. Hu, L. y Bentler, P. M. (1999). Cutoff criteria for fit indexes in covariance structure analysis: Conventional criteria versus new alternatives. Structural Equation Modelling, 6, 1-55. doi: 10.1080/10705519909540118. 
41. Jöreskog, K. G. y Sörbom, D. (1993). Structural equation modeling with the SIMPLIS command language. Chicago: Scientific Software International.

42. Kilpatrick, M., Hebert, E. y Jacobsen, D. (2002). Physical activity motivation. A practitioner's guido to self-determination theory. Journal of Physical Education, Recreation and Dance, 74(4), 36-41.

43. Kline, R. B. (2005), Principles and Practice of Structural Equation Modeling (2nd Edition ed.). New York: The Guilford Press.

44. Levy, R. y Hancock, G. R. (2007). A framework of statistical tests for comparing mean and covariance structure models. Multivariate Behavioral Research, 42, 33-66.

45. Ley 2/2006, de 3 de mayo, de Educación (BOE, no 106 del 4 de mayo de 2006).

46. Lim, B. S. C. y Wang, C. K. J. (2009). Perceived autonomy support, behavioural regulations in physical education and physical activity intention. Psychology of Sport and Exercise 10, 52-60. doi: 10.1016/j.psychsport.2008.06.003

47. Lonsdale, C., Sabiston, C. M., Raedeke, T. D., Ha, S. C. A. y Sum, K. W. R. (2009). Self-determined motivation and students' physical activity in PE classes and free-choice periods. Preventive Medicine, 48, 69-73. doi: 10.1016/j.ypmed.2008.09.013.

48. Matos, L. (2009). Adaptación de dos cuestionarios de motivación: Autorregulación del Aprendizaje y Clima de Aprendizaje. Revista Persona, $12,167-185$

49. Moreno, J. A. y Cervelló, E. (2003). Pensamiento del alumno hacia la Educación Física: su relación con la práctica deportiva y el carácter del educador. Enseñanza, 21, 345-362.

50. Moreno, J. A., Cervelló, E. y González-Cutre, D. (2007). Analizando la motivación en el deporte: un estudio a través de la teoría de la autodeterminación. Apuntes de Psicología, 25, 35-51.

51. Moreno, J. A., González-Cutre, D. y Ruiz, L. M. (2009a). Self-determined motivation and physical education importance. Human Movement, 10(1), 5-11.

52. Moreno, J. A. y Martínez, A. (2006). Importancia de la teoría de la autodeterminación en la práctica físico-deportiva: fundamentos e implicaciones prácticas. Cuadernos de Psicología del Deporte, 6(2), 39-54.

53. Moreno, J. A., Rodríguez, P. L. y Gutiérrez, M. (1996). Actitudes hacia la educación física: elaboración de un instrumento de medida. En Actas del III Congreso Nacional de Educación Física de Facultades de Educación y XIV de Escuelas Universitarias de Magisterio (pp. 507-516). Guadalajara: Universidad de Alcalá.

54. Moreno-Murcia, J. A., Huéscar, E. y Cervelló, E. (2012). Prediction of adolescents doing physical activity after completing secondary education. The Spanish Journal of Psychology, 15(1), 90-100. doi: http://dx.doi. org/10.5209/rev_SJOP.2012.v15.n1.37288

55. Moreno-Murcia, J. A., Sicilia, A., Cervelló, E., Huéscar, E. y Dumitru, D. (2011). The relationship between goal orientations, motivational climate and self reported discipline in physical education. Journal of Sports Science and Medicine, 10, 119-129.

56. Moreno-Murcia, J. A., Vera, J. A. y Del Villar, F. (2010). Search for autonomy in motor task learning in physical education university students. European Journal of Psychology of Education, 25, 37-47. doi: 10.1007/s10212-009-0008-7.

57. Moreno-Murcia, J. A., Zomeño, T., Marín, L. M., Ruiz, L. M. y Cervelló, E. (2013). Percepción de la utilidad e importancia de la educación física según la motivación generada por el docente. Revista de Educación, 362, 380-401. doi: 10.4438/1988-592X-RE-2011-362-165.

58. Moreno, J. A., Zomeño, T. E., Marín, L. E., Cervelló, E. y Ruiz, L. M. (2009b). Variables motivacionales relacionadas con la práctica deportiva extraescolar en estudiantes adolescentes de educación física. Apunts, Educación Física y Deportes, 95, 38-43.

59. Ntoumanis, N. (2005). A prospective study of participation in optional school physical education using a self-determination theory framework. Journal of Educational Psychology, 97, 444-453. doi: 10.1037/0022 0663.97.3.444

60. Núńez, J. L., Martín-Albo, J., Navarro, J. G. y González, V. M. (2006). Preliminary validation of a Spanish version of the Sport Motivation Scale. Perceptual and Motor Skills, 102, 919-930. doi: 10.2466/ PMS.102.3.919-930.

61. O’Neill, J. R., Pate, R. R. y Liese, A. D. (2011). Descriptive epidemiology of dance participation in adolescents. Research Quarterly for Exercise and Sport, 82(3), 373-380. doi: 10.1080/02701367.2011.10599769.

62. Pelletier, L. G., Fortier, M. S., Vallerand, R. J., Tuson, K. M., Brière, N. M. y Blais, M. R. (1995). Toward a new measure of intrinsic motivation, extrinsic motivation, and amotivation in sports: the Sport Motivation Scale (SMS). Journal of Sport and Exercise Psychology, 17, 35-53.

63. Rodríguez, P. L., García-Cantó, E., Sánchez-López, C. y López-Miñarro, P. A. (2013). Percepción de la utilidad de las clases de educación física y su relación con la práctica físico-deportiva en escolares. Cultura y Educación, 25(1), 65-76. doi: http://dx.doi.org/10.1174/113564013806309127.

64. Standage, M., Gillison, F. y Treasure, D. C. (2007). Intrinsic motivation and selfdetermination in exercise and sport. En M. S. Hagger, y N. L. D. Chatzisarantis (Eds.), Self-determination and motivation in physical education (pp. 71-85). Champaign, IL: Human Kinetics.

65. Standage, M., Gillison, F. B., Ntoumanis, N. y Treasure, D. (2012). Predicting Students' Physical Activity and Health-Related Well-Being: A Prospective Cross-Domain Investigation of Motivation Across School Physical Education and Exercise Settings. Journal of Sport y Exercise Psychology, 34, 37-60.

66. Su, Y. L. y Reeve, J. (2011). A meta-analysis of the effectiveness of intervention programs designed to support autonomy. Educational Psychology Review, 23, 159-188. doi: 10.1007/s10648-010-9142-7.

67. Taylor, I. M. y Lonsdale, C. (2010). Cultural differences in the relationships among autonomy support, psychological need satisfaction, subjective vitality, and effort in British and Chinese physical education. Journal of Sport \& Exercise Psychology, 32, 655-673.

68. Vallerand, R. J. (1997). Towards a hierarchical model of intrinsic and extrinsic motivation. En M. P. Zanna (Ed.), Experimental social psycho$\log y$ (pp. 271-361). New York: Academic Press.

69. Vlachopoulos, S. P. Karageorghis, C. I., y Terry, P. C. (2000). Motivation profiles in sport: A self-determination theory perspective. Research Quarterly for Exercise and Sport, 71, 387-397.

70. Williams, G. C. y Deci, E. L. (1996). Internalization of Biopsychosocial Values by Medical Students: A Test of Self-Determination Theory. Journal of Personality and Social Psychology, 70(4), 767-779. doi: 10.1037/0022-3514.70.4.767.

71. Williams, G. C., Grow, V. M., Freedman, Z. R., Ryan, R. M. y Deci, E. L. (1996). Motivational Predictors of Weight Loss and Weight-Loss Maintenance. Journal of Personality and Social Psychology, 70(1), 115126. 